\title{
Una Tautología en Hayek. El problema de la inasunción biológica en las Humanidades, I
}

\author{
Atafrascas Orbinio de la Estigia*
}

April 8, 2020

\begin{abstract}
The purpose of this brief note is to highlight the use of anthropocentric tautologies at the base of the argumentation of liberal ideology. Some of these authors, like Hayek, seem to consider that they are exempt from the general emptiness to which they are logically subject by the mere expedient of supposing them endowed with social or political meaning, without taking into account that human characteristics are carried by a natural biological entity that evolves, like the rest, by means of natural selection.
\end{abstract}

\section{Resumen}

El objetivo de esta breve nota es poner de manifiesto el uso de tautologías antropocéntricas en la base de la argumentación de la ideología liberal. Algunos de estos autores, como Hayek, parecen considerar que están exentas de la vacuidad general a la que están lógicamente sujetas por el mero expediente de suponerlas dotadas de significado social o político, sin reparar en que las características humanas las porta una entidad biológica natural que evoluciona, como el resto, por medio de la selección natural.

\section{Introducción}

Aparte de muchos otros logros y méritos en diversos ámbitos, en especial en Economía, se considera a Hayek como uno de los principales teóricos de las Ciencias Sociales del siglo XX (Véase, Vanberg, 2002 [17]; Caldwell, 2004 [4]; Postrel, 2004 [15]). A lo largo de su obra ha defendido el liberalismo clásico, el capitalismo, el libre mercado y la libertad del individuo, al tiempo que ha criticado, justamente, las ideologías socialistas o comunistas y las políticas de planificación o programación (Hayek, 1944 [6]).

Probablemente, la idea principal que recorre toda su obra filosófico-social, económica y política se refiere a la emergencia del orden natural espontáneo a partir de las decisiones de los individuos sujetos a reglas en un entorno idóneo (Hayek, 1967: 75 [7]). Parece clara la vinculación con la teoría evolutiva, en particular la darwinista. No obstante, Hayek ha dejado bien claro que la evolución de las reglas sociales ocurre de manera diferente de la evolución biológica, que simula (Hayek, 1988: 25 [9]); la evolución cultural es más rápida y lamarckiana, además de haber sido anterior y por tanto la semilla, y bien que insiste en ello, para las teorizaciones biológicas (Hayek, 1973: 23 [8]).

*Facultad de Filosofía A Fraja, Camiño Fondo, 7. Email: ng110777@gmail.com 


\section{Metodología}

Los pasos que seguiré se resumen en:

(i) Exposición del párrafo de Hayek.

(ii) Consecuencias que se derivan del párrafo tautológico hayekeano.

(iii) Explicación de la tautología en la selección natural sensu Popper y su analogía con la de Hayek.

(iv) Conclusión.

\section{$3 \quad$ El hecho}

Hayek, en su obra en tres volúmenes, 'Law, Legislation and Liberty' (Hayek, 1973), afirma:

"Society can thus exist only if by a process of selection rules have evolved which lead individuals to behave in a manner which makes social life possible."

(Hayek, 1978: 44 [8])

\subsection{Consecuencia}

Aunque Hayek se inspire en la famosa exposición aristotélica sobre la natural y teleológica preeminencia, superioridad o anterioridad del estado y la comunidad sobre el individuo (Aristóteles, libro I, capítulo I [1]), el párrafo hayekeano aparenta ser un error de carácter lógico nada desdeñable. En efecto, no es difícil armonizar de forma equivalente el término 'society' con la expresión 'social life'. La vida social posible es justo la que únicamente se produce en sociedad. Además, la sociedad no tiene sentido si no estuviese conformada por los individuos que la componen. La tautología es diáfana. Probablemente, Hayek consideró que, si bien los resultados de las acciones individuales sujetas a reglas son impredecibles en detalle, es necesario establecer una diferencia con respecto a una perspectiva más general, la de la sociedad en su conjunto, pues, pese a todo, es factible constituir hormas generales que subsuman el fin proléctico de todo el sistema. De otra forma, que si conociésemos la exacta relación entre el orden de las acciones de los individuos y el orden de las reglas que regulan su conducta, entonces podríamos toparnos con resultados variables, y falsables, que nos indicarían cuáles de las reglas nos coartasen y debiésemos limitar o eliminar y cuáles nos permitirían mejorar y evolucionar (Hayek, 1967 [7]). Esto es, según Aristóteles, o brutos o dioses.

Empero, este razonamiento es una martingala, pues deja en el aire cómo se ejecutaría esa relación epistemológica entre acciones y nomoi, dado que el carácter ontológico de ambas no acepta una categorización homogénea inmediata ¿Por qué se produce este error de raigambre aristotélica en Hayek? Porque cree que es posible eludir el problema lógico soslayando sus implicaciones racionales al igual que hizo Popper con el caso evolutivo sosteniendo la inexistencia de leyes históricas y olvidando a sabiendas la Ciencia, la Biología. 


\subsection{Popper y el problema tautológico en evolución}

Uno de sus amigos vieneses fue Karl Popper. Hayek cita a menudo a Popper; veintiocho veces en 'Law, Legislation and Liberty', y tiene en cuenta buena parte de sus razonamientos relacionados con el descubrimiento y la demarcación científicos y la concepción popperiana de una sociedad abierta ¿Por qué Hayek se apoya en Popper respecto del asunto que trata esta nota? Popper, en 'Objective Knowledge', afirmó, un año antes de la publicación de 'Law, Legislation and Liberty', que la teoría evolutiva darwinista era tautológica, o casi, que la expresión que define la selección natural como 'the survival of the fittest' es equivalente a sustituir 'fittest' por 'those that survive' y tendríamos entonces la huera expresión de que sobreviven los que sobreviven (Popper, 1972: 241$242[14])^{1}$. Tras recibir todo tipo de críticas por su incomprensión de la teoría evolutiva y el mecanismo de la selección natural (por ejemplo, Stamos, 1996 [16]; Hull, 1999 [10]; Diéguez, 2004 [5]; Boldachev, 2014 [2]), en 1978 el mismo Popper reconoció su 'culpabilidad' al interpretar mal los asertos biológicos, por supuesto descargando, nada humildemente, esa culpa en que había sido influenciado por otros biólogos eminentes que habían cometido el mismo error (Popper, 1978: 344 [13]). Reconoce ahí también que la teoría de la selección natural está muy lejos de ser tautológica (Popper, 1978: 346 [13]). Cabalmente, Hayek consideró que su afirmación, de lejanos tintes aristotélicos, sobre la conformación de las sociedades podía escapar de la culpa tautológica al igual que la selección natural. Como se mencionó más arriba, siguiendo a Aristóteles y Popper, por el simple artefacto intelectual de no fijarse en los detalles individuales impredecibles y apelar a una generalización comprehensiva que fundase una sociedad mejorada, liberal.

\section{3 coda}

Recordemos, finalmente, que esta perspectiva liberal es también dependiente de lo que Popper señaló sobre cualquier epistemología, esto es, que debe analizarse desde su génesis e historia por un lado y su verdad, validez y justificación, por otro (Popper, 1972: 67 [14]). Sin embargo, la verdad, validez o justificación de la argumentación liberal descansa, a tenor de lo expuesto, en tautologías que la pervierten, por más que podamos retrotraernos hasta autores desde los que anclar falacias de autoridad.

No se trata pues de teorías falsables, al decir de Popper (1976: 195 [12]), sino de meros programas metafísicos de investigación; no hablamos de ciencia, antes bien de marcos heurísticos que la faciliten, que permitan construir verdaderas teorías científicas, si se da el caso, si es posible el caso. Conversamente, para que el liberalismo se erija en una ideología fundamentada en proposiciones racionales debe rechazar el auxilio de los argumentos circulares, falaces o infalsables y optar por reconocer que si pretende hablar del hombre, del Homo sapiens, previamente deberá bajar hasta la procelosa Biología para no desdeñar conocimientos bien establecidos y obliterar los obvios decursos publicados, so riesgo de volverse una posición dogmática. En esta tesitura, la tautología de Hayek podría pasar, ser

\footnotetext{
${ }^{1}$ Naturalmente, otros autores posteriores han insistido en que la definición de selección natural es tautológica o que requiere ulteriores precisiones para definirla con mayor rigor ( $C f$. Hung, 2014 [11])
} 
salvada por la denominada 'lógica situacional' (Popper, 1976: 195 [12]), mas carece de la entidad empírica necesaria. Como dice Bueno refiriéndose a autores como Mises, Hayek o Milton Friedman, los liberales "no disponen de una teoría científica del liberalismo económico", ya que éste está basado en "una doctrina lisológica de la libertad humana individual", por tanto tiene "más de ideología metafísica que de ciencia positiva" (Bueno, 2015 [3]). Y es que la argumentación económico-liberal de Hayek funda un irracionalismo ético que hace depender la elección humana de la aceptación de su concepción de sociedad de mercado (Vergara, 2012 [18]). Su desconocimiento de la realidad biológica le permite escribir sin preocuparse de la necesaria coincidencia con lo que es el caso, como a tantos discípulos pasados, actuales y youtubers rallando su tibieza, iluminados quién sabe si sólo por la luz de los focos. A menudo, como a Hayek, sucede esto, cuando nuestras teorías filosóficas, con cualquier adjetivación, se envuelven cual erizo porque en lontananza se nos antoja claro el fin buscado, ignorando lo que ya se sabe en disciplinas científicas.

Comoquiera que la argumentación de Hayek se basa en última instancia en la aparición de un cierto orden natural espontáneo y éste surge a partir de las decisiones de los individuos que deben obedecer normas que los obligan y facultan a la vez para la construcción de una sociedad y que este proceso se fundamenta en una simple tautología, deberemos concluir que el entramado filosófico social o político de tintes liberales está transido de circularidad. Hayek está en contra del intervencionismo y el proteccionismo estatal, en contra de toda planificación que diluya la capacidad de elección individual; sin embargo, su afirmación da pábulo a esos mismos mecanismos, pues ¿qué tipo de reglas deben ser las que posibiliten la eutaxia social liberal? ¿No serán aquéllas heterónomas, las dadas por la estructura comunitaria para todo aquel que alcanza su capacidad supuestamente libre de elección?

\section{Bibliografía}

[1] Aristóteles. Política. Trad. Patricio de Azcárate, Obras filosóficas de Aristóteles. Madrid 1873-1875, 10 volúmenes. Imprenta de la Biblioteca de Instrucción y Recreo, Rubio 25, Madrid, 302 pp., 1873. Disponible en línea en: http://www.filosofia.org/cla/ari/azc03.htm.

[2] Boldachev, Alexander. Natural Selection or Problem Solving. Critical Reevaluation of Karl Poppper's Evolutionism. Studia Humana Vol. 3(3): 29 ?42 pp., 2014.

[3] Bueno, Gustavo. Rasguños: El liberalismo como ideal humanístico. El Catoblepas. Revista crítica del presente, 161: 2, 2015. Disponible en línea en: http://nodulo.org/ec/2015/n161p02.htm

[4] Caldwell, Bruce. Hayek's Challenge. An intellectual biography of F.A. Hayek. The University of Chicago Press, Chicago, 489 pp., 2004.

[5] Diéguez, Antonio. Popper como filósofo de la biología. In W. J. González (ed.), Karl Popper: Revisión de su legado. Madrid, Unión Editorial, 413-450 pp., 2004. 
[6] Hayek, F.A. The Road to Serfdom. Text and Documents. The Definitive Edtion. The collected works of F.A. Hayek, vol. II (Bruce Caldwell ed.). The University of Chicago Press, 283 pp., [1944] 2007.

[7] Hayek F.A. Studies in Philosophy, Politics and Economics. University of Chicago Press, Chicago, 314 pp., 1967.

[8] Hayek, F.A. Law, legislation and Liberty. A new statement of the liberal principles of justice and political economy. Vol. 1 Rules and Order. Routledge. London, 188 pp., [1973] 2003.

[9] Hayek, F.A. The fatal Conceit. The Errors of Socialism. The collected works of F.A. Hayek, vol. I (W. W. Bartley ed.). The University of Chicago Press, 283 pp., 1988.

[10] Hull, David L. The Use and Abuse of Sir Karl Popper. Biology and Philosophy, 14: 481-504, 1999.

[11] Hunt T. Reconsidering the logical structure of the theory of natural selection. Communicative $\mathcal{E}$ integrative biology, 7(6), e972848, 2014.

[12] Popper, Karl. Unended quest. An intellectual autobiography. Taylor and Francis e-Library: Routledge. New edition with Postscript and updated bibliography, London and New York, 315 pp., [1976] 2005.

[13] Popper, Karl. Natural Selection and the Emergence of Mind. Dialectica, vol. 32, no. 3/4, 339-355 pp., 1978.

[14] Popper, Karl R. Objective Knowledge: An Evolutionary Approach. Oxford University Press Inc. Revised edition, 395 pp., [1972] 1979.

[15] Postrel, V. Friedrich the Great. The Boston Globe, 11 January, 2004.

[16] Stamos, David N. Popper, Falsifiability, and Evolutionary Biology. Biology and Philosophy, 11: 161-191, 1996.

[17] Vanberg, V. Hayek. Friedrich A von (1899-1992). In International Encyclopedia of the Social \& Behavioral Sciences, 6482-86. [2002] 2015.

[18] Vergara Estévez, Jorge. La ética de Friedrich von Hayek. 2012: 159-181 pp. In González Meyer, R. \& Richards H. (comp.) Hacia otras economías. Crítica al paradigma dominante. Biblioteca Digital DIBRI-UCSH por Universidad Católica Silva Henríquez. 2012. 